\title{
Risk Factors and Outcomes of Postoperative Catheter-Associated Urinary Tract Infection in Colorectal Surgery Patients: A Retrospective Cohort Study
}

\author{
Ali H. Farsi ${ }^{1}$ \\ 1. Department of Surgery, Faculty of Medicine, King Abdulaziz University, Jeddah, SAU
}

Corresponding author: Ali H. Farsi, farsiresearch@gmail.com

\section{Abstract \\ Introduction}

Catheter-associated urinary tract infection (CAUTI) is a relatively common cause of postoperative morbidity in colorectal surgery patients. It has been associated with increased length of stay and mortality.

\section{Methods}

We performed a retrospective cohort study of 620 colorectal surgeries to assess the prevalence of CAUTI and its relationship with preoperative and operative factors. We also sought to identify its association with postoperative outcomes.

\section{Results}

We found that CAUTI occurred in $20.6 \%$ of colorectal procedures. We found that CAUTI was associated with older patient age, female gender, higher BMI, higher American Society of Anesthesiologists (ASA) classification, lower hemoglobin, higher creatinine, lower albumin, urgent procedures, bilateral ureteric stent placement, usage of double-J (DI) stents, postoperative abdominal sepsis, and perioperative steroid usage. CAUTI was also associated with the presence of underlying medical conditions such as hypertension, ischemic heart disease, chronic kidney disease, cerebrovascular disease, and diabetes. With regards to postoperative outcomes, it was associated with postoperative stroke, myocardial infarction, prolonged length of stay, Intensive care unit stay, and mortality.

\section{Conclusion}

CAUTI remains a significant cause of morbidity in colorectal patients. Our patient population had a significantly higher risk of CAUTI compared to other series. Though sometimes labelled a minor postoperative complication, its occurrence is associated with other more significant postoperative

Review began 04/29/2021 Review ended 05/12/2021 Published 05/19/2021

\section{() Copyright 2021}

Farsi. This is an open access article distributed under the terms of the Creative Commons Attribution License CC-BY 4.0., which permits unrestricted use, distribution, and reproduction in any medium, provided the original author and source are credited.
Categories: Urology, General Surgery, Infectious Disease

Keywords: urinary tract infection, colorectal surgery, ureteric catheterization, urinary catheter, surgical outcomes

\section{Introduction}

Catheter-associated urinary tract infections (CAUTI) are one of the most common hospital-acquired infections [1], with colorectal surgery patients being at particular risk [1,2]. They are often considered minor complications but remain a significant cause of morbidity as they are associated with an increased postoperative length of stay (PLOS) and increased hospital cost. CAUTI is also associated with an increased risk of mortality [2-4]. They are also believed to contribute to other morbidities, such as venous thromboembolism, pneumonia, and delirium in older medical patients by limiting mobility [5].

Colorectal surgery patients often require urinary tract instrumentation in order to place ureteric catheters to guard against ureteric injury during surgery, or Foley catheters to enable urine output measurement intraoperatively and postoperatively and to avoid postoperative urinary retention [6].

In this study we aimed to identify the rates and frequency of CAUTI among colorectal surgery patients and the association of CAUTI with other adverse postoperative events.

\section{Materials And Methods}

\section{Setting}


We obtained ethical approval from the Unit of Biomedical Ethics Research Committee at the Faculty of Medicine, King Abdulaziz University (reference no 263-21). We performed a retrospective cohort study by reviewing the operating room list from January 2013 to January 2020 at King Abdulaziz University Hospital, an academic tertiary care center, to identify all colorectal surgeries.

\section{Inclusion and exclusion criteria}

Our inclusion criteria were adult patients over the age of 18 undergoing surgery on the colon and rectum. Our exclusion criteria were patient whose surgery did not require entry into the peritoneal cavity such as stomal revision or transanal excision and patients who did not have a Foley catheter placed. If a patient had more than one procedure, we excluded the second procedure if it occurred within six months of the first procedure. We did this in order to eliminate any surgeries related to complications from index procedure such as an operation to treat an anastomotic leak, evisceration, early stoma complications, and so forth.

\section{Data collection and outcomes of interest}

We reviewed the patients' charts and extracted baseline characteristics including age, gender, body mass index (BMI), American Society of Anesthesiologists (ASA) classification, medical history, including hypertension, diabetes, ischemic heart disease (IHD), heart failure, chronic liver disease (CLD), chronic kidney disease (CKD), and asthma. We extracted the patients' preoperative laboratory values, including hemoglobin, white blood cell count (WBC), platelets, international normalized ratio(INR), activated partial thromboplastin time (aPTT), albumin and creatinine. We reviewed the operative note to extract operative details such as procedure urgency, duration, procedure type, method of surgery and the use of ureteric catheters. We reviewed the postoperative course for details about postoperative outcomes such as severe postoperative intraabdominal sepsis (e.g., anastomotic leak, missed bowel injury, and rectal stump leak), urine culture results, Intensive care unit admission, stroke, myocardial infarction (MI), and mortality that occurred within the 30 days postoperative or until discharge. We defined CAUTI as the presence of a positive urine culture postoperatively in a patient with symptoms suggestive of urinary tract infection or sepsis. Our primary outcomes of interest were the incidence and the predictors of CAUTI among colorectal surgery patients. Our secondary outcome was the association of CAUTI with other adverse postoperative events.

\section{Statistical methods}

Descriptive statistics were used to calculate durations, means and standard deviation. Binominal logistic regression (univariate and multivariate regression) was used to test the predictors of the binary outcome variables. Shapiro-Wilk test was used to test the normality of the study samples. Simple frequency tables, cross-tabulations, and percentages were calculated. Linear Regression was used to test the predictors of the continuous outcome variable postoperative length of stay. Chi-square test was used to test and describe the relation between two categorized variables. Independent sample t-test analysis was applied to determine whether there was statistical evidence that the mean difference between two groups was significant or not significant. The level $\mathrm{p}<0.05$ was used as the cut-off value for significance. We performed statistical analysis using SPSS statistics for windows version 20.0 (IBM Corp., Armonk, NY, USA).

\section{Results}

Out of 750 patients who underwent surgery on the colon and rectum, 130 had one or more exclusion criteria. We thus included 620 patients. In our study population, $55.3 \%$ were males, $29.2 \%$ were diabetic, $38.7 \%$ had urgent procedures, and $17.2 \%$ required at least one ureteric catheter to be placed. The full demographics, clinical and operative details of the included patients can be found in Table 1 .

\begin{tabular}{|c|c|c|c|c|}
\hline Table 1 & & $\mathbf{n}$ & Percent \% & Mean \pm SD \\
\hline Age at operation (years) & & & & $53.99 \pm 16.9$ \\
\hline \multirow[t]{2}{*}{ GENDER } & Male & 343 & 55.3 & \\
\hline & Female & 277 & 44.7 & \\
\hline $\mathrm{BMI} \mathrm{Kg} / \mathrm{m}^{2}$ & & & & $26.18 \pm 6.23$ \\
\hline \multirow[t]{3}{*}{ smoking history } & Non-smoker & 333 & 82.8 & \\
\hline & Ex-smoker & 20 & 5.0 & \\
\hline & Smoker & 49 & 12.2 & \\
\hline \multirow[t]{2}{*}{ Diabetes } & Not diabetic & 439 & 70.8 & \\
\hline & Diabetic & 181 & 29.2 & \\
\hline Hypertension & No & 417 & 67.3 & \\
\hline
\end{tabular}




\section{Cureus}

\begin{tabular}{|llll} 
& Yes & 203 & 32.7 \\
Ischemic heart disease & No & 567 & 91.5 \\
Cerebrovascular disease & Yes & 53 & 8.5 \\
& No & 596 & 96.1 \\
Chronic kidney disease & Yes & 24 & 3.9 \\
ASA classification & No & 574 & 92.6 \\
& Yes & 46 & 7.4 \\
& I & 43 & 6.9 \\
& II & 283 & 45.7 \\
& III & 235 & 38.0 \\
& IV & 55 & 8.9 \\
& V & 3 & .5
\end{tabular}

Hemoglobin

WBC

Creatinine

Preop Albumen level

$11.29 \pm 2.36$

$8.13 \pm 5.45$

$90.73 \pm 81.44$

$30.15 \pm 8$

Preop International Normalized Ratio

$1.1 \pm 0.21$

Activated Partial Thromboplastin Time

$32.69 \pm 10.02$

Procedure Urgency

Routine

61.3

Emergency

$240 \quad 38.7$

Regional Anesthesia

No

$245 \quad 39.5$

Laparoscopic/Open Procedure

Yes

$375 \quad 60.5$

Laparoscopic

$179 \quad 28.9$

Rectal Surgery

Open

$441 \quad 71.1$

No

$487 \quad 78.5$

Yes

$133 \quad 21.5$

Preoperative Length of Stay (days)

$5.66 \pm 11.36$

Intraoperative PRBC Transfusion (units)

None

67.7

1

9.0

3

Type of Urinary Catheter

Latex

$30 \quad 5.1$

Number of Ureteric Catheters

$\begin{array}{lcc}\text { Silicon } & 559 & 94.9 \\ \text { None } & 513 & 82.7 \\ \text { Single } & 33 & 5.3 \\ \text { Bilateral } & 74 & 11.9\end{array}$




\section{Cureus}

Regular Ureteric Catheter/Double-J

$\begin{array}{lcc}\text { None } & 513 & 82.9 \\ \text { DJ } & 39 & 6.3 \\ \text { Catheter } & 67 & 10.8\end{array}$

\section{TABLE 1: Patient demographics and baseline laboratory investigations}

ASA: American Society of Anesthesiologists; WBC: white blood cells; PRBC: packed red blood cells

Out of 620 patients, 128 (20.6\%) developed CAUTI. Factors that were associated with postoperative CAUTI in univariate analysis included older age, female gender, higher BMI, ASA class IV and V, lower hemoglobin, higher creatinine, lower albumin, higher WBC count, higher activated partial thromboplastin time (aPTT), urgent procedures, bilateral ureteric place placement, usage of double-J (DJ) stents, presence of postoperative abdominal sepsis, and perioperative steroid usage. Certain underlying medical conditions were also found to be associated with CAUTI, such as the presence of hypertension, ischemic heart disease, chronic kidney disease, cerebrovascular disease, and diabetes, Factors that were associated with CAUTI in multivariate analysis included lower hemoglobin, bilateral ureteric catheter placement, and postoperative abdominal sepsis. Full details of the univariate and multivariate analysis can be found Table 2 .

\begin{tabular}{|c|c|c|c|c|c|c|c|c|c|}
\hline \multirow{3}{*}{ Table 2} & & \multicolumn{4}{|c|}{ Univariant Analysis } & \multicolumn{4}{|c|}{ Multivariate Analysis } \\
\hline & & \multirow{2}{*}{ OR } & \multicolumn{2}{|c|}{ 95\% C.I. } & \multirow{2}{*}{ p-value } & \multirow{2}{*}{ OR } & \multicolumn{2}{|c|}{ 95\% C.I. } & \multirow{2}{*}{$\mathrm{p}$-value } \\
\hline & & & Lower & Upper & & & Lower & Upper & \\
\hline Age & & 1.02 & 1.01 & 1.03 & .001 & 1.01 & 0.99 & 1.03 & .284 \\
\hline \multirow[t]{2}{*}{ GENDER } & Male & (Ref) & & & & & & & \\
\hline & Female & 1.66 & 1.12 & 2.46 & .011 & 1.63 & 0.86 & 3.11 & .135 \\
\hline BMI & & 1.03 & 1.00 & 1.06 & .035 & 1.00 & 0.95 & 1.04 & .845 \\
\hline \multirow{3}{*}{ Smoking History } & Non-smoker & (Ref) & & & & & & & \\
\hline & Ex-smoker & 0.87 & 0.28 & 2.70 & .816 & 0.86 & 0.23 & 3.14 & .815 \\
\hline & Smoker & 0.31 & 0.11 & 0.89 & .030 & 0.36 & 0.11 & 1.18 & .092 \\
\hline \multirow[t]{2}{*}{ Hypertension } & No & (Ref) & & & & & & & \\
\hline & Yes & 1.98 & 1.33 & 2.95 & .001 & 1.54 & 0.75 & 3.18 & .241 \\
\hline \multirow{2}{*}{ Ischemic Heart Disease } & No & (Ref) & & & & & & & \\
\hline & Yes & 1.76 & 0.94 & 3.27 & .076 & 1.30 & 0.46 & 3.67 & .615 \\
\hline \multirow[t]{2}{*}{ Chronic Kidney Disease } & No & (Ref) & & & & & & & \\
\hline & Yes & 3.00 & 1.61 & 5.60 & .001 & 2.09 & 0.61 & 7.19 & .242 \\
\hline \multirow[t]{2}{*}{ Diabetes } & No & (Ref) & & & & & & & \\
\hline & Yes & 2.28 & 1.52 & 3.42 & .000 & 1.06 & 0.54 & 2.09 & .873 \\
\hline \multirow[t]{2}{*}{ Cerebrovascular disease } & No & (Ref) & & & & & & & \\
\hline & Yes & 2.89 & 1.25 & 6.68 & .013 & 0.73 & 0.16 & 3.41 & .692 \\
\hline \multirow[t]{5}{*}{ ASA Classification } & 1 & (Ref) & & & & & & & \\
\hline & II & 1.36 & 0.51 & 3.65 & .540 & 2.31 & 0.27 & 19.90 & .445 \\
\hline & III & 2.49 & 0.94 & 6.63 & .068 & 3.69 & 0.42 & 32.66 & .240 \\
\hline & IV & 4.34 & 1.47 & 12.82 & .008 & 2.91 & 0.26 & 32.26 & .384 \\
\hline & V & 15.20 & 1.16 & 199.63 & .038 & 3.11 & 0.05 & 177.36 & .582 \\
\hline Preoperative Hemoglobin & & 0.89 & 0.82 & 0.97 & .010 & 1.17 & 1.01 & 1.36 & .037 \\
\hline
\end{tabular}




\section{Cureus}

\begin{tabular}{|c|c|c|c|c|c|c|c|c|c|}
\hline \multicolumn{2}{|c|}{ Preoperative White blood cell count } & 1.04 & 1.01 & 1.07 & .022 & 1.00 & 0.93 & 1.08 & .967 \\
\hline \multicolumn{2}{|l|}{ Preoperative Albumin } & 0.95 & 0.93 & 0.97 & .000 & 0.95 & 0.90 & 1.00 & .057 \\
\hline \multicolumn{2}{|l|}{ Preoperative Creatinine } & 1.00 & 1.00 & 1.00 & .026 & 1.00 & 1.00 & 1.01 & .495 \\
\hline \multicolumn{2}{|l|}{ Preoperative INR } & 1.60 & 0.70 & 3.68 & .263 & & & & \\
\hline \multicolumn{2}{|l|}{ Preoperative aPTT } & 1.03 & 1.01 & 1.04 & .006 & 1.03 & 0.99 & 1.06 & .109 \\
\hline \multicolumn{2}{|l|}{ Preoperative Length of Stay } & 1.01 & 1.00 & 1.03 & .114 & & & & \\
\hline \multirow[t]{2}{*}{ Procedure Urgency } & Routine & (Ref) & & & & & & & \\
\hline & Emergency & 1.59 & 1.08 & 2.36 & .020 & 0.89 & 0.41 & 1.96 & .775 \\
\hline \multirow[t]{2}{*}{ Regional Anasthesia } & No & (Ref) & & & & & & & \\
\hline & Yes & 0.74 & 0.50 & 1.10 & .133 & & & & \\
\hline \multirow[t]{2}{*}{ Rectal Surgery } & No & (Ref) & & & & & & & \\
\hline & Yes & 1.44 & 0.92 & 2.25 & .115 & & & & \\
\hline \multirow[t]{2}{*}{ Procedure Type } & Laparoscopic & (Ref) & & & & & & & \\
\hline & Open & 1.50 & 0.95 & 2.36 & .083 & 1.81 & 0.91 & 3.59 & .090 \\
\hline \multirow[t]{3}{*}{ U stent which side } & None & (Ref) & & & & & & & \\
\hline & Single & 1.91 & 0.88 & 4.15 & .101 & 1.28 & 0.36 & 4.55 & .702 \\
\hline & Bilateral & 1.98 & 1.16 & 3.41 & .013 & 2.66 & 1.12 & 6.32 & .026 \\
\hline \multirow[t]{3}{*}{ Ureteric catheter used } & None & (Ref) & & & & & & & \\
\hline & Double J & 3.06 & 1.56 & 6.02 & .001 & 2.43 & 0.77 & 7.69 & .132 \\
\hline & Regular Catheter & 1.50 & 0.83 & 2.71 & .184 & 2.43 & 0.77 & 7.69 & .132 \\
\hline \multirow[t]{2}{*}{ Urethral Catheter used } & Latex & & & & & & & & \\
\hline & Silicon & 0.85 & 0.36 & 2.03 & .716 & & & & \\
\hline \multicolumn{2}{|l|}{ Intraop PRBCs transfusion } & 1.17 & 0.95 & 1.45 & .137 & & & & \\
\hline \multirow[t]{2}{*}{ Perioperative Steroid use } & No & (Ref) & & & & & & & \\
\hline & Yes & 1.66 & 1.09 & 2.53 & .019 & 1.33 & 0.66 & 2.66 & .423 \\
\hline \multirow[t]{2}{*}{ Postop Abdominal Sepsis } & No & (Ref) & & & & & & & \\
\hline & Yes & 2.90 & 1.63 & 5.18 & .000 & 2.95 & 1.23 & 7.07 & .015 \\
\hline
\end{tabular}

\section{TABLE 2: Univariate and multivariate analysis}

ASA: American Society of Anesthesiologists; INR: international normalized ratio; aPTT: activated partial thromboplastin time; PRBCs: packed red blood cells

With regards to postoperative outcomes, we found that CAUTI was associated with increased risk of intensive care unit (ICU) stay, myocardial infarction (MI), stroke, and mortality in the first 30 days postoperatively. The details can be found in Table 3 . 


\section{Cureus}

\begin{tabular}{|c|c|c|c|c|}
\hline \multirow[b]{2}{*}{ Postop complication } & \multirow{2}{*}{$\mathrm{p}$-value } & \multirow{2}{*}{ Odd Ratio (OR) } & \multicolumn{2}{|c|}{$95 \% \mathrm{Cl}$ for OR } \\
\hline & & & Lower & Upper \\
\hline Postop ICU stay & $<0.001$ & 2.675 & 1.793 & 3.993 \\
\hline Postop MI & .001 & 2.411 & 1.414 & 4.112 \\
\hline Postop stroke & .030 & 3.407 & 1.125 & 10.322 \\
\hline Postop 30-day mortality & .006 & 2.445 & 1.298 & 4.607 \\
\hline
\end{tabular}

\section{TABLE 3: Postoperative outcomes}

ICU: intensive care unit; MI: myocardial infarction

Using linear regression, we found that CAUTI was a significant predictor for increased postoperative length of stay, with the mean PLOS increased by 11 days for those who had CAUTI (the mean PLOS for those without CAUTI was 15.06 [SD 20.11], and for those who developed CAUTI 26.06 [SD 28.07]) $(\mathrm{p}<0.001$ and $\mathrm{R}=$ $0.20)$. Of note, postoperative length of stay was not normally distributed.

\section{Discussion}

In this study, we found that $20.6 \%$ of patients undergoing colorectal surgery in our hospital population developed CAUTI. We found that CAUTI was associated with several adverse postoperative outcomes such as MI, stroke, PLOS, admission to the ICU, and mortality.

The risk of CAUTI was significantly elevated in our population compared to other studies that found it to be 4.1\%-7.7\% in colorectal surgery patients $[1,2,6,7]$. We hypothesize that this maybe due to several factors. We included emergency procedures, while many series assessed only elective surgeries. Many studies have been performed at specialized centers where poorly educated and noncompliant patients maybe underrepresented. Diabetes, a significant predictor of CAUTI in our and other series, was highly prevalent in our patient population (29.2\%), as about $19.6 \%$ of Saudis have it [8]. Our institution recently initiated an Enhanced Recovery After Surgery (ERAS) protocol, and most of the patients in our study preceded this protocol. The ERAS protocols promote earlier removal of urinary catheters. Though we did not assess duration of catheterization in our study population, it has been shown previously that CAUTI increases by 5$10 \%$ per catheter-day after the first 48 hours of catheterization [9].

We noted several associations between CAUTI and patient characteristics, lab values, and operative details. Some factors such as patient age, gender, ASA score, steroid use, diabetes, hypertension, and a history of cerebrovascular disease have been previously identified as risk factors for CAUTI after colorectal surgery $[1,2,4]$. Others such as preoperative hemoglobin, albumin, WBC count, hypertension, CKD, and emergency surgery have been identified as risk factors for morbidity and mortality after colorectal surgery in general $[7,10,11]$. Some of these factors, such as WBC and aPTT, are more likely to be abnormal in an emergency situation, for which prolonged catheterized maybe necessary. We also found it to be associated with postoperative abdominal sepsis (which we defined as anastomotic leak, missed enterotomy, and rectal stump leakage). We believe it is probably related to the need for prolonged catheterizations due to the development of these significant postoperative complication. We found a history of smoking to be inversely related to the risk of CAUTI. This maybe due to underreporting of smoking history, which could affect the validity of this result.

We found no association between CAUTI and IHD, BMI, regional anesthesia, type of surgery (laparoscopic vs open), and rectal surgery. This is despite other studies having found an association with some of these factors $[1,4]$.

We found that the type of Foley catheter was not associated with CAUTI. This is consistent with previous studies. A Cochrane review found no difference between CAUTI risk among standard Foley catheters. It did note a reduction in the risk of UTI and bacteriuria with silver alloy and antibiotic-impregnated Foley catheters, respectively [12]. We found that the presence, number, and type of ureteric stent was associated with the risk of CAUTI. In our institution, when a surgeon requests preoperative placement of a ureteric catheter, the type and size of ureteric catheter placed is left to the discretion of the urologist. It has been documented that DJ stents are associated with an increased risk of bacteriruria [13]. Additionally, vesicoureteric reflux has been noted in patients with DJ stents [14]. This combination could potentially lead to increased risk of acute pyelonephritis and sepsis [15] in patients who develop CAUTI, as when placed 
preoperatively in the setting of colorectal surgery, they will often remain in place until they can be removed electively later. We did not assess if patients with CAUTI and DJ stents had a worse outcome than those without DJ stents, and this maybe an avenue for further research. If this were found to be true, then it would suggest that simple ureteric catheters should be chosen over DJ stents.

We found that CAUTI was associated with increased risk of ICU admission, MI, stroke, increased length of stay, and 30-day mortality [4]. This is similar to the increased risk of overall morbidity associated with CAUTI $[4,16]$. This may be multifactorial. Indwelling catheters limit mobility, potentially leading to an increased incidence of venous thromboembolism (VTE) [5]. Other research has shown that CAUTI is associated with the development of systemic sepsis in $3.6 \%$ of patients [9]. It may also occur that CAUTI can develop secondary to the requirement for prolonged catheterization from another postoperative morbidity, such as a stroke or unplanned reintubation.

An additional concern with the development of CAUTI in the postoperative period is that it can have longterm effects. Previous research that assessed the effect of postoperative CAUTI on survival after major surgery (of any type) found that the occurrence of a UTI was associated with a mortality rate of $6 \%$ at 30 days, $25 \%$ at one year, and $58 \%$ at five years [17]. It has been shown that infectious postoperative complications in colorectal cancer patients is associated with decreased long-term survival [18]. Potential reasons could be that infectious complications affect cell mediated immunity, leading to increased risk of cancer recurrence, or those who develop significant complications are less likely to receive adjuvant therapy [18].

Many methods, some of them at minimal cost, have been found to reduce the risk of CAUTI. These include daily electronic prompts to remove the Foley catheter, implementation of an ERAS protocol, usage of closed catheter drainage systems, minimizing disconnection of the catheter junction, placement of the urine bag below the level of the bladder, and consideration of usage of antimicrobial-coated urinary catheters, among others. Prophylactic antibiotics for CAUTI are not recommended [16, 19, 20]. Some authors have suggested routine suprapubic catheters in abdominal surgery instead of Foley catheters to decrease the risk of CAUTI, but an randomized controlled trial (RCT) showed no difference in risk of CAUTI [21].

\section{Limitations}

Our study was a retrospective study. Our study sample is on the smaller side compared to other studies that have assessed postoperative CAUTI. We did not assess the duration of catheterization, an important factor that predicts the occurrence of CAUTI, as this information was not readily available to us. We did not assess the association of CAUTI with prolonged operative time. Though we have shown that CAUTI is associated with other postoperative complications, it is difficult to determine if it is a risk factor for them or the other adverse events lead to a longer catheterization time and thus an increased risk of CAUTI.

\section{Conclusions}

Catheter-associated urinary tract infection is a significant cause of morbidity after colorectal surgery. Our patient population had a significantly higher risk of CAUTI compared to other series. Attempts to identify the underlying reasons for this higher CAUTI risk and steps to reduce it at an institutional level are essential. Though sometimes labelled a minor postoperative complication, the occurrence of CAUTI is associated with other more significant postoperative complications, including death. A new finding in our study was the association between the risk of CAUTI with bilateral ureteric catheterization and DJ stents in colorectal surgery. Future research should look into the superiority of one method of ureteric instrumentation over another in reducing the risk of urinary tract infection in a randomized setting.

\section{Additional Information}

\section{Disclosures}

Human subjects: Consent was obtained or waived by all participants in this study. Unit of Biomedical Ethics Research Committee at the Faculty of Medicine, King Abdulaziz University issued approval 263-21. We obtained ethical approval from the Unit of Biomedical Ethics Research Committee at the Faculty of Medicine, King Abdulaziz University(reference no 263-21). Animal subjects: All authors have confirmed that this study did not involve animal subjects or tissue. Conflicts of interest: In compliance with the ICMJE uniform disclosure form, all authors declare the following: Payment/services info: All authors have declared that no financial support was received from any organization for the submitted work. Financial relationships: All authors have declared that they have no financial relationships at present or within the previous three years with any organizations that might have an interest in the submitted work. Other relationships: All authors have declared that there are no other relationships or activities that could appear to have influenced the submitted work.

\section{Acknowledgements}

The author would like to thank the following for their invaluable contribution. Professor Hasan Farsi, Dr Alaa Shabkah, Dr Nora Trabulsi, Dr Abdullah Sultan, Dr Anfal Nawawi, Dr Abeer Samman, Renad Bakhsh, Ibtisam 
Gasm, Muruj Alkhamisi, Hind Alsulami, Malak Bajnaid, Morouj Fatani, Jood Simbawa, Ahlam Hadri, and Joud Salloom.

\section{References}

1. Regenbogen SE, Read TE, Roberts PL, Marcello PW, Schoetz DJ, Ricciardi R: Urinary tract infection after colon and rectal resections: more common than predicted by risk-adjustment models. J Am Coll Surg. 2011, 213:784-92. 10.1016/j.jamcollsurg.2011.08.013

2. Attaluri V, Kiran RP, Vogel J, Remzi F, Church J: Risk factors for urinary tract infections in colorectal compared with vascular surgery: a need to review current present-on-admission policy? J Am Coll Surg. 2011, 212:356-61. 10.1016/j.jamcollsurg.2010.11.014

3. Dimick JB, Chen SL, Taheri PA, Henderson WG, Khuri SF, Campbell DA Jr: Hospital costs associated with surgical complications: a report from the private-sector National Surgical Quality Improvement Program. J Am Coll Surg. 2004, 199:531-7. 10.1016/j.jamcollsurg.2004.05.276

4. Sheka AC, Tevis S, Kennedy GD: Urinary tract infection after surgery for colorectal malignancy: risk factors and complications. Am J Surg. 2016, 211:31-9. 10.1016/j.amjsurg.2015.06.006

5. Holroyd-Leduc JM, Sen S, Bertenthal D, et al.: The relationship of indwelling urinary catheters to death, length of hospital stay, functional decline, and nursing home admission in hospitalized older medical patients. J Am Geriatr Soc. 2007, 55:227-33. 10.1111/j.1532-5415.2007.01064.x

6. Kin C, Rhoads KF, Jalali M, Shelton AA, Welton ML: Predictors of postoperative urinary retention after colorectal surgery. Dis Colon Rectum. 2013, 56:738-46. 10.1097/DCR.0b013e318280aad5

7. Alves A, Panis Y, Mathieu P, Mantion G, Kwiatkowski F, Slim K: Postoperative mortality and morbidity in French patients undergoing colorectal surgery: results of a prospective multicenter study. Arch Surg. 2005, 140:278-83, discussion 284. 10.1001/archsurg.140.3.278

8. Whiting DR, Guariguata L, Weil C, Shaw J: IDF diabetes atlas: global estimates of the prevalence of diabetes for 2011 and 2030. Diabetes Res Clin Pract. 2011, 94:311-21. 10.1016/j.diabres.2011.10.029

9. Wald HL, Ma A, Bratzler DW, Kramer AM: Indwelling urinary catheter use in the postoperative period: analysis of the national surgical infection prevention project data. Arch Surg. 2008, 143:551-7. 10.1001/archsurg.143.6.551

10. Longo WE, Virgo KS, Johnson FE, et al.: Risk factors for morbidity and mortality after colectomy for colon cancer. Dis Colon Rectum. 2000, 43:83-91. 10.1007/BF02237249

11. Masoomi H, Kang CY, Chen A, Mills S, Dolich MO, Carmichael JC, Stamos MJ: Predictive factors of inhospital mortality in colon and rectal surgery. J Am Coll Surg. 2012, 215:255-61.

10.1016/j.jamcollsurg.2012.04.019

12. Schumm K, Lam TB: Types of urethral catheters for management of short-term voiding problems in hospitalised adults. Cochrane Database Syst Rev. 2008, CD004013. 10.1002/14651858.CD004013.pub3

13. Farsi HM, Mosli HA, Al-Zemaity MF, Bahnassy AA, Alvarez M: Bacteriuria and colonization of doublepigtail ureteral stents: long-term experience with 237 patients. J Endourol. 1995, 9:469-72. 10.1089/end.1995.9.469

14. Mosli HA, Farsi HM, al-Zimaity MF, Saleh TR, al-Zamzami MM: Vesicoureteral reflux in patients with double pigtail stents. J Urol. 1991, 146:966-9. 10.1016/s0022-5347(17)37976-4

15. Chen SJ, Huang CP, Chiu KY, et al.: Association of acute pyelonephritis with double-J ureteral stenting: a nationwide population-based case control study. Scand J Urol. 2021, 55:61-6. 10.1080/21681805.2020.1817142

16. Nagle D, Curran T, Anez-Bustillos L, Poylin V: Reducing urinary tract infections in colon and rectal surgery . Dis Colon Rectum. 2014, 57:91-7. 10.1097/DCR.0000000000000019

17. Khuri SF, Henderson WG, DePalma RG, Mosca C, Healey NA, Kumbhani DJ: Determinants of long-term survival after major surgery and the adverse effect of postoperative complications. Ann Surg. 2005, 242:32641; discussion 341-3. 10.1097/01.sla.0000179621.33268.83

18. Artinyan A, Orcutt ST, Anaya DA, Richardson P, Chen GJ, Berger DH: Infectious postoperative complications decrease long-term survival in patients undergoing curative surgery for colorectal cancer: a study of 12,075 patients. Ann Surg. 2015, 261:497-505. 10.1097/SLA.0000000000000854

19. Hooton TM, Bradley SF, Cardenas DD, et al.: Diagnosis, prevention, and treatment of catheter-associated urinary tract infection in adults: 2009 International Clinical Practice Guidelines from the Infectious Diseases Society of America. Clin Infect Dis. 2010, 50:625-63. 10.1086/650482

20. Miller TE, Thacker JK, White WD, et al.: Reduced length of hospital stay in colorectal surgery after implementation of an enhanced recovery protocol. Anesth Analg. 2014, 118:1052-61. 10.1213/ANE.0000000000000206

21. Baan AH, Vermeulen H, van der Meulen J, Bossuyt P, Olszyna D, Gouma DJ: The effect of suprapubic catheterization versus transurethral catheterization after abdominal surgery on urinary tract infection: a randomized controlled trial. Dig Surg. 2003, 20:290-5. 10.1159/000071693 